\title{
Génération d'harmoniques d'ordre élevé dans les gaz : résultats récents
}

\author{
P. Antoine, B. Carré, M. Gaarde*, M. Lewenstein, A. L'Huillier*, A. Persson* et \\ P. Salières
}

Service des Photons, Atomes et Molécules, Bâtiment 522, CEA, Centre d'Études de Saclay, 91191 Gif-sur-Yvette cedex, France

* Department of Physics, Lund Institute of Technology, 22100 Lund, Suède

\begin{abstract}
Résumé : La génćration d'lanrmoniques d'ordre élevé dans les gaz est une source de rayonnement UVX aux caractéristiques originales - courte durée. bonne colérence temporelle et spatiale. grande brillance. L'étude de la polarisation du rayonnement constitue un test sévère du modèle théorique. Elle complète la caractérisation des harmoniques produites à partir d'une seule fréquence fondamentale. Nous montrons que l'on peut aussi produire des harmoniques partiellement accordables. à partir de deux frén̨uences fondanentales dont l'une est accordable.
\end{abstract}

La génération d'harmoniques d'ordre élevé dans les gaz constitue une source de rayonnement UV et $X$ mous aux caractéristiques intéressantes, complémentaires de celles des autres sources comme le rayonnement synchrotron, le laser à électrons libres, le laser $\mathrm{X}$ ou le rayonnement $X$ produit par plasma. Depuis plusieurs années, le groupe de Saclay développe l'étude des aspects fondamentaux de la génération d'harmoniques dans les gaz rares.

\section{CARACTERISTIQUES DU RAYONNEMENT HARMONIQUE D'ORDRE ELEVE}

Nous rappelons les principales caractéristiques du rayonnement harmonique produit auprès de la chaine laser femtoseconde du CEA-DRECAM à Saclay (laser pulsé Ti :Sa, impulsions de $150 \mathrm{fs}$, énergie par impulsion $50 \mathrm{~mJ}$, taux de répétition $20 \mathrm{~Hz}$ ). Le rayonnement fondamental à $790 \mathrm{~nm}$ est focalisé à l'aide d'une longue focale $(1 \mathrm{~m})$ dans un jet pulsé de gaz rare (pression $\approx 10$ Torr). Les éclairements utiles varient, selon le gaz, entre $10^{14}$ et $10^{15} \mathrm{Wcm}^{-2}$. Des spectres typiques du rayonnement harmonique produit dans $\mathrm{Xe}, \mathrm{Ar}$ et $\mathrm{Ne}$, respectivement, sont donnés en figure I. Ils sont constitués, pour des raisons de symétrie, des multiples impairs de la fréquence fondamentale. Les spectres présentent une allure caractéristique : après une décroissance rapide dans les premiers ordres (qui n'apparaissent pas sur la figure), le nombre de photons est sensiblement constant dans une plage de fréquence appelée plateau (qui correspond, dans les conditions des spectres en figure 1 , à $q \leq 17(\mathrm{Xe}), \mathrm{q} \leq 23(\mathrm{Ar}), \mathrm{q} \leq 53(\mathrm{Ne})$ ), limitée par une coupure où le nombre de photons décroît rapidement. Dans $\mathrm{Ne}$, le plateau peut s'étendre jusqu'à $170 \mathrm{eV}$. Le nombre optimisé de photons émis par impulsion est estimé en figure 2. Il varie de $10^{11}$ à $20 \mathrm{eV}$ (Xe) à moins de $10^{7}$ au-delà de $40 \mathrm{eV}(\mathrm{Ne}$ ). La courte durée des impulsions et la bonne cohérence spatiale du rayonnement permettent en principe, après refocalisation, d'atteindre des éclairements de $10^{14} \mathrm{Wcm}^{-2}(20 \mathrm{eV})$ à $10^{10} \mathrm{Wcm}^{-2}(40 \mathrm{eV})$

Les applications de ce rayonnement commencent à voir le jour; elles doivent tirer parti des ses caractéristiques originales

- La courte durée des impulsions (50-120 fs), leur synchronisation naturelle avec le rayonnement fondamental, trouvent des applications dans les expériences de type « pompe+sonde » résolues en temps, en physique atomique, moléculaire et des solides.

- les éclairements étevés permettent d'envisager l'étude de phénomènes non linéaires.

- la lumière est polarisée linéairement quand l'efficacité de conversion est maximale et l'on fait facilement tourner cette polarisation. 
- La grande cohérence temporelle $(\Delta v \Delta \tau \approx 1)$ et spatiale (source équivalente à une source ponctuelle et faisceau quasi-gaussien) donne accès à l'interférométrie UVX.

- le rayonnement harmonique est partiellement accordable.

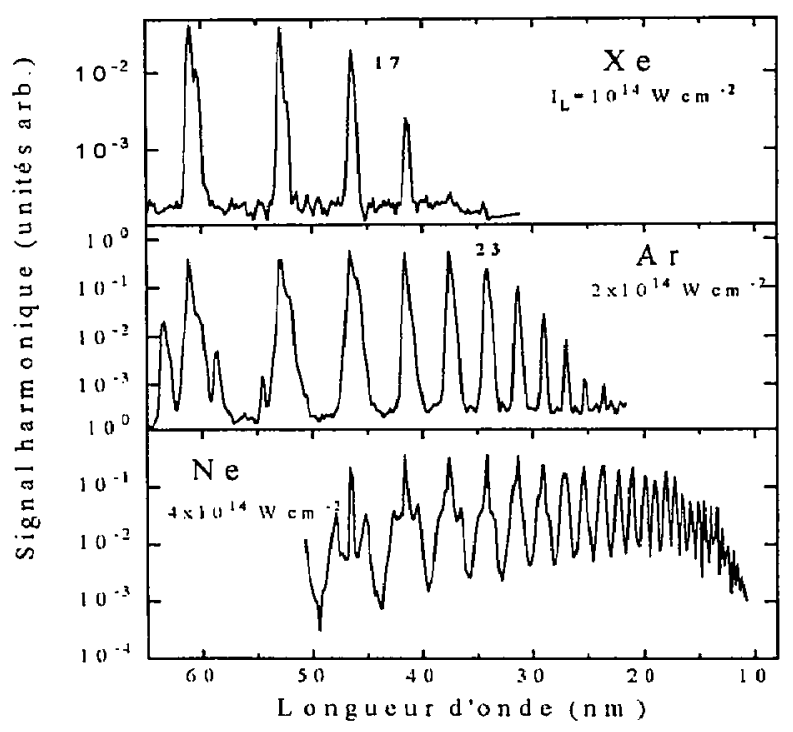

Figure 1: Spectres harmoniq̨ues dans le xénon. l'argon et le nćon, montrant la distribution d'intensité plateau/coupure

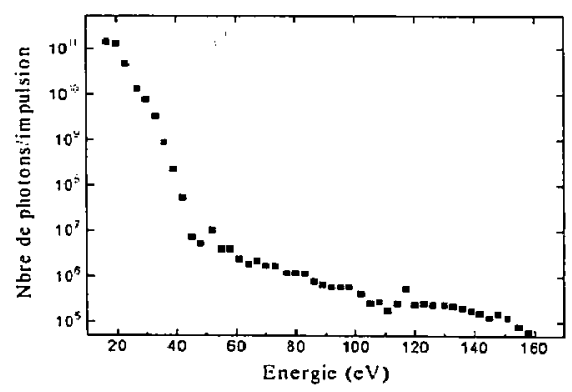

Figure 2 : Nombre de plotons harmoniques produits par impulsion, en utilisant différents gaz (Xe, Ar, Ne)

\section{MODELISATION DE LA GENERATION D'HARMONIQUES D'ORDRE ELEVE}

La génération d'harmoniques d'ordre élevé est aujourd'hui bien comprise théoriquement, sous les deux aspects, étroitement liés, de la réponse de l'atome unique et de la propagation du rayonnement harmonique dans le milieu. 
Il est bien connu que les harmoniques les plus basses (ordre $3,5,7$ ) sont produites par un mécanisme perturbatif, impliquant les états excités liés de l'atome. Dans ce cas, le nombre de photons harmoniques décroît rapidement avec l'ordre de non linéarité. Au contraire, les harmoniques d'ordre élevé sont produites par un mécanisme non perturbatif, comme l'indique l'existence d'un plateau dans le spectre. L'interprétation semi-classique [1] de la génération d'harmoniques d'ordre élevé permet de se faire une idée simple du processus. Elle distingue 2 étapes dans la réponse de l'atome unique (la polarisation du fondamental est ici supposée linéaire): i) un électron de valence à l'état fondamental passe par effet tunnel à travers la barrière de potentiel de largeur finie, somme du potentiel coulombien et de l'énergie potentielle dans le champ laser (considéré comme statique pendant l'ionisation tunnel); ii) l'électron oscille librement dans le champ; après avoir gagné de l'énergie cinétique, il peut, $\mathrm{s}^{\prime} \mathrm{il}$ repasse au voisinage du coeur ionique, se recombiner en émettant un photon harmonique. L'énergie maximale que l'électron peut acquérir dans le champ détermine, avec le potentiel d'ionisation de l'atome, la limite du plateau. Ce modèle très simple conduit à une loi de coupure qui est en bon accord avec les mesures. Selon ce modèle, on modifie le processus de génération d'harmoniques en affectant la trajectoire de l'électron; la dépendance de la génération d'harmoniques vis-à-vis de la polarisation fondamentale est une illustration de ce principe que nous développons dans la suite.

Le modèle quantique de la réponse de l'atome unique, développé par $\mathrm{M}$. Lewenstein et coll. [2], fonde rigoureusement l'interprétation semi-classique dans un régime de champ fort et de basse réquence. Ce modèle calcule le dipôle atomique, ses composantes de Fourier d'ordre $q$, qui sont les termes source non linéaires de l'émission de l'harmonique q $\omega$.

Une partie importante de la modélisation consiste finalement à propager les différents champs harmoniques dans le milieu macroscopique en présence des termes source non linéaires. Le champ harmonique se construit de façon efficace s'il y a un bon accord de phase entre les champs microscopiques émis par les différents points du milieu. Les études ont montré que la phase, par rapport au champ fondamental, du moment dipolaire harmonique (composante d'ordre q du dipôle atomique) joue un rôle majeur dans l'établissement de cet accord. Il existe ainsi des conditions pour lesquelles le rayonnement émis présente une grande cohérence temporelle mais aussi spatiale [3].

Nous présentons dans la suite deux études expérimentales récentes, d'une part de la polarisation du rayonnement harmonique, d'autre part de la génération d'harmoniques à partir de deux fréquences fondamentales.

\section{POLARISATION DU RAYONNEMENT HARMONIQUE}

L'étude de la polarisation complète la caractérisation du rayonnement harmonique. Elle permet la comparaison poussée entre mesures et simulations, la polarisation étant une grandeur particulièrement sensible aux paramètres de l'interaction. L'interprétation semi-classique permet de comprendre que la génération d'harmoniques est maximale pour une polarisation linéaire du fondamental, et qu'elle décroît rapidement quand la polarisation devient elliptique $[4,5]$. Dans ce dernier cas, l'électron ne repasse plus près du cceur avec lequel il ne peut plus se recombiner; la génération d'harmoniques n'est permise que par l'extension transverse du paquet d'onde électronique. Pour une polarisation fondamentale linéaire, la polarisation harmonique est linéaire et de même direction; une lame de phase dans le faisceau fondamental suffit à en contrôler l'orientation. Pour une polarisation fondamentale d'ellipticité $\varepsilon_{\mathrm{f}}$ (rapport petit axe/grand axe), repérée par l'orientation $\phi_{r} d u$ grand axe, la polarisation harmonique est localement elliptique. En général, pour des ordres élevés (en régime non perturbatif), son 
ellipticité diffère de celle du fondamental, ainsi que son orientation : la polarisation harmonique a «tourné » par rapport à la polarisation fondamentale, le sens de rotation dépendant du caractère droit ou gauche de celle-ci. Par ailleurs, la polarisation harmonique varie avec l'éclairement du fondamental, du fait des variations du module et la phase du dipôle atomique ; elle n'est donc pas uniforme, ni dans le temps - pendant la durée de l'impulsion - ni dans l'espace - suivant la dimension radiale transverse du faisceau fondamental - . En conséquence, le rayonnement harmonique «moyen», grandeur intégrée que l'on mesure, n'est que partiellement polarisé. Un rayonnement partiellement polarisé (pp) est caractérisé à l'aide des paramètres de Stokes, $s_{0}, s_{1}, s_{2}, s_{3}$ [6]. En termes de ces paramètres, il se décompose formellement en un champ non polarisé (np) et un champ complètement polarisé (cp), ayant en tout point et à tout instant une polarisation d'ellipticité et d'orientation données. Le degré de polarisation, noté $P$, est le rapport de l'éclairement moyen du champ polarisé (cp) à celui du champ total (pp). Par définition, on appelle ellipticité $\varepsilon_{\mathrm{h}}$ et orientation $\phi_{11}$ du champ (pp) les grandeurs associées au champ (cp), qui s'expriment en fonction des paramètres de Stokes.

Dans notre étude, nous avons étendu aux harmoniques d'ordre élevé les résultats de Weihe et al [7], relatifs à des ordres faibles. Nous avons caractérisé les variations de $\varepsilon_{\mathrm{h}}$ et $\phi_{\mathrm{h}}$ en fonction de l'ellipticité $\varepsilon_{\mathrm{f}}$ du fondamental ( $\phi_{\mathrm{h}}$ mesure dans la suite l'orientation relative de la polarisation harmonique par rapport à la polarisation fondamentale). Expérimentalement, pour chaque orientation de la polarisation fondamentale d'ellipticité donnée $\varepsilon_{f}$, on mesure l'intensité harmonique transmise par un polariseur simplement constitué d'un miroir Ag à l'incidence de $45^{\circ}$. On mesure ainsi les paramètres $s_{0}, s_{1}, s_{2}$ associès au champ harmonique. Le paramètre $s_{3}$, qui mesure le «taux » de polarisation circulaire, n'est pas accessible : sa mesure demande d'utiliser l'équivalent d'une lame quart-d'onde dans l'UVX, dispositif difficile à élaborer. L'orientation $\phi_{\mathrm{h}}$ de la polarisation harmonique est donnée par $\operatorname{tg} 2 \phi_{\mathrm{h}}=\frac{s_{2}}{s_{1}}$; ainsi définie, $\phi_{\mathrm{h}}$ s'interprète bien comme la moyenne, pondérée par l'éclairement harmonique, de l'orientation de la polarisation harmonique. A l'aide des paramètres $s_{1}, s_{2}$, on détermine une borne supérieure de $\varepsilon_{\mathrm{h}}$ (pour déterminer la valeur exacte de $\varepsilon_{\mathrm{l}}$, il est nécessaire de connaître $s_{3}$ ). L'interprétation physique de $\varepsilon_{1}$ est plus difficile : $\varepsilon_{\mid 1}$ n'est pas une moyenne pondérée de l'ellipticité du champ harmonique et ne caractérise celle-ci qu'indirectement ( ${ }^{\prime}$ ).

Nous avons mesuré systématiquement l'angle de rotation $\phi_{h}$ et la borne sup. de l'ellipticité $\varepsilon_{\mathrm{h}}$, pour les harmoniques $17(\mathrm{Xe}, \mathrm{Ar}, \mathrm{Ne}), 23(\mathrm{Ar}, \mathrm{Ne}), 29$ et $33(\mathrm{Ne})$. Les mesures sont effectuées, pour chaque gaz, dans les conditions de la figure 1. Pour une harmonique donnée, nous mesurons la variation de la polarisation en fonction du gaz, de l'éclairement fondamental, et plus spécialement de la position de l'harmonique par rapport à la coupure du spectre. Les résultats pour les harmoniques 17 et 23 sont rapportés en figure 3 et 4 . Quand $\mathrm{H} 17$ est proche de la coupure (fig. $3 \mathrm{Xe}$ ), l'ellipticité (borne sup.) reste faible, très inférieure à celle du fondamental. La rotation de la polarisation n'excède pas $5^{\circ}$ (fig. $4 \mathrm{Xe}$ ). Au contraire, quand H17 s'éloigne de la coupure (Ar puis Ne), l'ellipticité devient importante. La rotation de la polarisation atteint $40^{\circ}$ dans le néon (fig. $4 \mathrm{Ne}$ ). La même observation peut être faite pour H23.

\footnotetext{
' Si on pose $\operatorname{tg} \%=\varepsilon_{\text {h. }}$ alors $\sin 2 \chi=\frac{s_{3}}{\sqrt{s_{1}^{2}+s_{2}^{2}+s_{3}^{2}}}$. Soit $\operatorname{tg} \psi_{1}$ l'clliplicité locale du champ harmonique, alors la moyenne, pondérée par l'éclaireınent du champ larmonique. de la quantié $\sin 2 \psi_{1} \operatorname{s}^{\prime}$ écrit $\left\langle\sin 2 \chi_{1}\right\rangle=P \sin 2 \chi$, où $P$ est le degré de polarisation.
} 


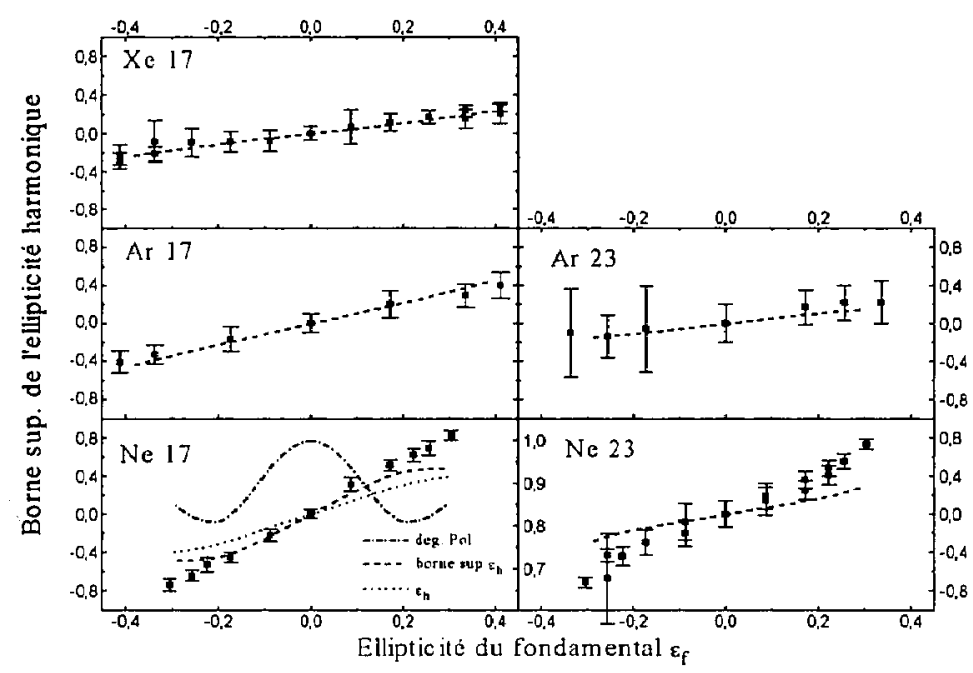

Figure 3 : Borne supćricure de l'cllipticité $\varepsilon_{\mid}$des harınoniques 17 et 23 dans Xe, Ar et Ne, mesurée et calculée (---). Dans Ne. on compare la borne sup. à l'ellipticilé exacie $\varepsilon_{\mathrm{l}}$ calculée (...) : les quantités différent d'autant plus que le degré de polarisation $\mathrm{P}$ s'éloigne de l (.--.. : éclielle de droite).

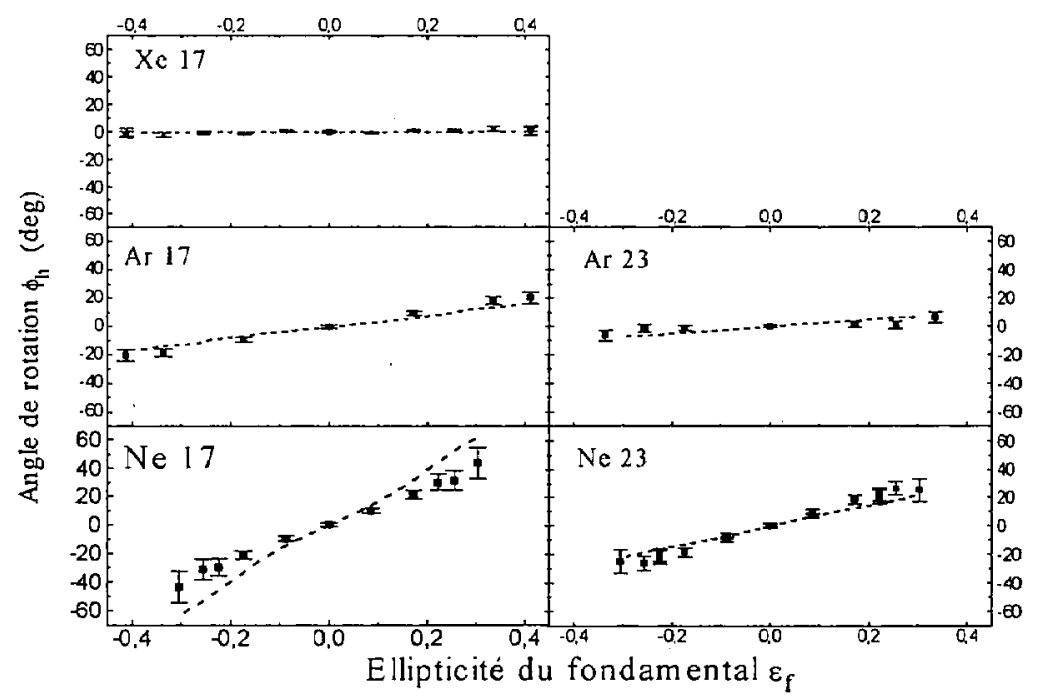

Figure 4: Angle de rotation $\phi_{h}$ de la polarisation des harmoniques 17 et 23 par rapport à la polarisation fondamentale, dans Xe, Ar el Ne, mesuréc et calculée (---).

On note également que pour un gaz donné ( $\mathrm{Ar}, \mathrm{Ne}$ ), l'ellipticité et la rotation de la polarisation sont d'autant plus marquées que l'harmonique est éloignée de la coupure, c'est-à- 
dire d'ordre faible. Il apparaît donc que la position relative à la coupure détermine la polarisation de l'harmonique.

L'accord entre les angles mesurés et calculés est excellent. De même, la borne sup de l'ellipticité est très bien reproduite dans la simulation. Il faut rappeler que le calcul, successivement, du dipôle atomique en polarisation elliptique, du champ harmonique après propagation, des paramètres de Stokes associés, est fait sans paramètre ajustable. Le bon accord obtenu nous fonde à considérer le calcul de $\varepsilon_{\mathrm{h}}$ (qui n'est pas mesurée) comme correct. On note que $\varepsilon_{h}$ reste inférieure à l'ellipticité du fondamental. La cifférence entre $\varepsilon_{\mathrm{h}}$ et sa borne sup., visible sur la figure 3 ( $\mathrm{Ne} \mathrm{17),} \mathrm{est} \mathrm{d'autant} \mathrm{plus} \mathrm{grande} \mathrm{que} \mathrm{le} \mathrm{degré} \mathrm{de} \mathrm{polarisation} \mathrm{P}$ s'éloigne de 1 .

L'étude ci-dessus montre que l'on peut caractériser la polarisation du rayonnement harmonique, expérimentalement (presque complètement) et théoriquement. Le caractère polarisé, ainsi que la facilitè à orienter la polarisation, rend les harmoniques propres à différentes applications.

\section{GENERATION D'HARMONIQUES A PARTIR DE DEUX FREQUENCES}

Eichmann et al [8] ont montré que l'on peut générer des harmoniques « accordables » à partir de deux fréquences fondamentales, $\omega_{1}$ fixe, $\omega_{2}$ variable mais de faible éclairement. On obtient une lumière harmonique de fréquence $p_{1} \omega_{1}+p_{2} \omega_{2}\left(p_{1}+p_{2}\right.$ impair), variable selon le domaine d'accord de $\omega_{2}$. Dans notre étude [9], nous avons étendu les résultats publiés, $p_{1} \leq 10$ et $p_{2}= \pm 1$, à des ordres élevés, $p_{1} \leq 48$ et $p_{2}= \pm 1, \pm 2$. Nous montrons que l'on peut produire, par ce mécanisme, un rayonnement partiellement accordable jusqu'à $60 \mathrm{eV}$.

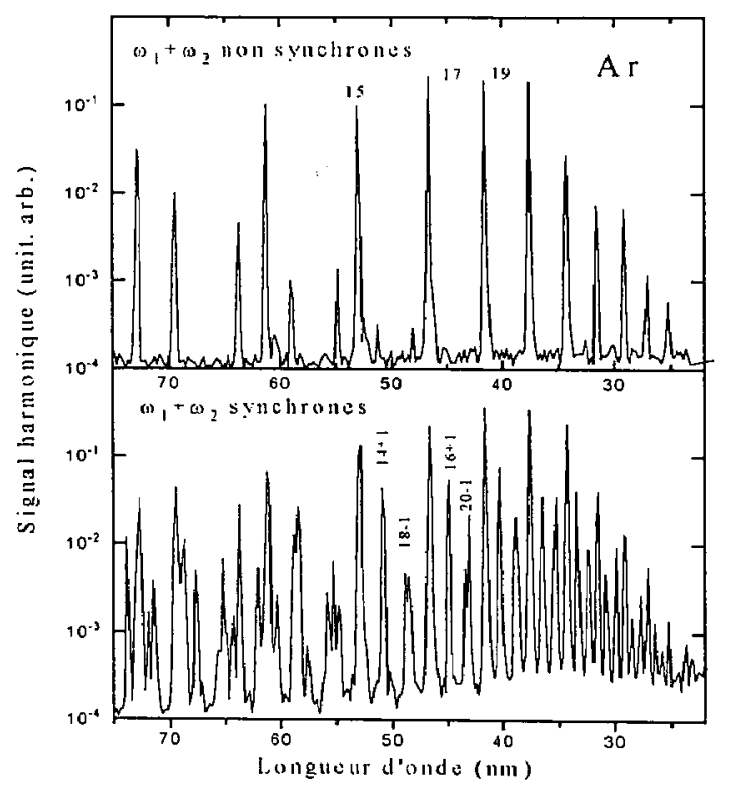

Figure 5 : Specires harmoniques mesurés dans l'argon en présence de deux fréquences $\omega_{1}$ et $\omega_{2}$ a) non synchrones et b) sỵnclirones, les structures additionnelles correspondent aux harmoniques $p_{1} \omega_{1} \pm \omega_{2}$. 
Un rayonnement accordable autour de $500 \mathrm{~nm}$ (fréquence $\omega_{2}$ ), d'énergie $100 \mu \mathrm{J}$, est produit à partir d'un oscillateur paramétrique amplifié (OPA ; le visible est produit comme la somme de l'IR à $790 \mathrm{~nm}$ et du signal OPA à $1360 \mathrm{~nm}$ ). Il est superposé spatialement au rayonnement IR (fréquence $\omega_{l}, 10 \mathrm{~mJ}$ ) dans le jet de gaz rare. La figure 5 montre les spectres d'harmoniques mesures dans l'argon, quand les deux rayonnements sont respectivement a) décalés dans le temps, b) synchrones. Dans le premier cas, les harmoniques sont produites à partir de la seule fréquence $\omega_{1}$, l'éclairement à $\omega_{2}$ étant trop faible. Dans le cas synchrone, on observe plusieurs structures intermédiaires correspondant aux harmoniques $p_{1} \omega_{1}+p_{2} \omega_{2}$, avec $p_{1} \leq 28$ et $p_{2}= \pm 1$ dans l'argon, $p_{1} \leq 48$ et $p_{2}= \pm 1$ dans le néon. Les ordres $p_{2}= \pm 1, \pm 2$ ont été observés dans le xénon. Compte tenu de la faible cohérence spatiale du faisceau OPA et de la mauvaise focalisation qu'elle permet, le rapport des éclairements $I_{O P A} / I_{R}$ n'est que de $10^{-4}$ environ. Même dans ces conditions, l'éclairement des harmoniques à 2 couleurs peut atteindre $10 \%$ de celui des harmoniques d'ordre voisin $\left(p_{1} \pm 1\right) \omega_{1}$. La figure 6 compare le nombre de photons pour les processus $\left(p_{1}+1\right),\left(p_{1}\right)+1$ et $\left(p_{1}\right)-1$ (les nombres absolus de photons n'ont pas été optimisés).

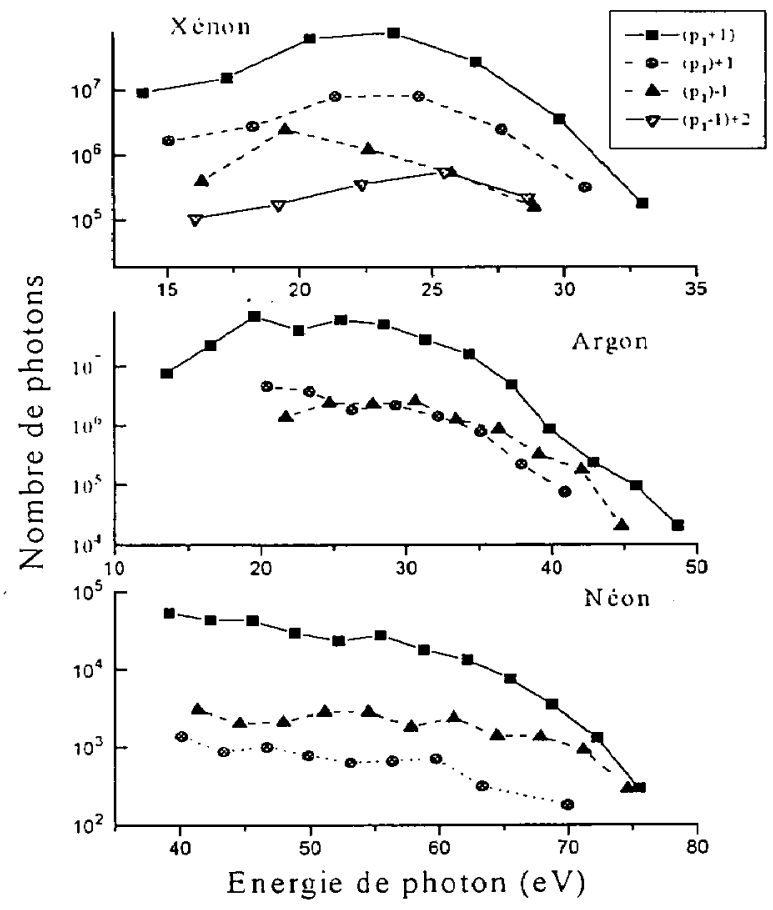

Figure 6 : Comparaison des nombres de photons (non optimisćs) entre les processus à une couleur $\left(\mathrm{p}_{1}+1\right) \omega_{1}$; à deux couleurs. $p_{1}\left(\theta_{1}+\omega_{2}=\right.$ el $p_{1}\left(\omega_{1}-\omega_{2}\right.$ ) dans $X c$. Ar et $N e$ : $p_{1}\left(\omega_{1}+2 \omega\right)$ dans $X e$. Le nombre de photons $p_{1} \omega_{1}+\omega_{2}$ et $p_{1} \omega_{1}-()_{2}$ attcint $10 \%$ du nombre de photons $\left(p_{1}+1\right) \omega_{1}$.

On note que le processus $\left(p_{1}\right)+1$ domine $\left(p_{1}\right)-1$ aux ordres faibles $(X e)$. Les deux processus sont de même importance au voisinage de l'ordre $25(\mathrm{Ar}, 39 \mathrm{eV})$; ( $\left.\mathrm{p}_{1}\right)-1$ devient 
dominant aux ordres élevés. La variation relative des nombres de photons a été reproduite dans un modèle, où l'on calcule le dipôle atomique en présence des deux champs. Elle reflète la dynamique de l'électron qui diffère notablement entre les cas +1 et -1 .

\section{Références :}

[1] Kulander K. C., Sclaifer K. J.. Krause J. L, "Super-Intense Laser-Atom Plysics ", B. Piraux, A. L'Huillier et K. Rzazcwski Eds.. NATO ASI scries B 316 (Plcnum Press, New York 1993). p. 95.

Corkum P. B.. Phys. Rev. Lett. 71 (1993) 1994.

[2] Lewenstein M.. Balcou P., Ivanov M. Y.. L'Huillicr A.. Corkum P. B., Phys. Rev. A, 49 (1994) 2117.

[3] Salières P., L'Huillicr A.. Lewenstcin M.. Phys. Rev. Lett. 74 (1995) 3776.

Lewenstein M.. Salières P., L'Huillier A.. Phys. Rev' A 52 (1995) +747.

[4] Budil K. S. Salières P.. L'Huillier A.. Ditmire T. Perry M. D.. Phyv. Rev. 4 48 (1993) R3437.

Liang Y.. Amonosov M. V.. Chin S. L.. J. Phus. B 27 (19\%4) 1296.

Dielrich P.. Burnell N. H. Wanor M. Y., Corkun P. B.. Phvs. Rey, A 50 (1995) R3585.

Burnat N. H. Kan C. Corkum P. B.. Phus. Rev. 451 (1995) R3418.

[5] Antoine P.. L'Huillier A.. Lewenstein M. Salic̀res P.. Carré B., Ihys. Rev. A 53 (1996) 1725.

[6] Born M.. Wolr E. Principles of Opties. 6" edition (Pergamon Press. New York. 1964) p.544.

[7] Weile F. A.. Dutta S. K.. Korn G.. Du D.. Bucksbaum P. H.. Shkolnikov P. L.. Phus. Rev. A 51 (1995) R3433.

[8] Eichmann H.. Meyer S.. Ripcl K. Momma C. Wellegelauscn B., Phys. Rev. A1 50 (1994) R2834.

Eichunann H., Egberl A.. Nolte S.. Momma C.. Wellegelausen B.. Bccker W. Long S.. McIver J. K., Phys. Rey. A 51 (1995) R3414.

[9] Gaarde M.. Antoine P.. Persson A.. Carré B., L'Huillier A.. Walıströ̀n C.-G.. J. Phys. B 29 (1996) L163. 